\title{
RECHERCHES BIOMÉTRIQUES SUR LA COULEUR DES ABEILLES CARNIOLIENNES EN YOUGOSLAVIE (Apis mellifica var. carnica Polum.)
}

\author{
J. RIHAR
}

Institut Agronomique de Slovénie, Ljubljana, Yougoslavie.

\section{SOMMAIRE}

L'abeille carniolienne est considérée comme une abeille franchement noire. L'auteur procède à un examen systématique de la coloration du $\mathrm{z}^{\mathrm{e}}$ tergite abdominal des abeilles de son pays et montre que beaucoup d'abeilles carnioliennes présentent des marques jaunes sur cette partie de leur corps. Elles n'ont cependant jamais l'extension des marques colorées que présente l'abeille italienne.

\section{INTRODUCTION ETT POSITION DU PROBLÈME}

Il est essentiel pour le travail de sélection des abeilles de trouver des caractères morphologiques assez spécifiques pour permettre de distinguer des populations d'abeilles d'origines génétiques différentes. Dans un territoire où la race est unique, comme en Slovénie, où j'ai pris la plupart des échantillons, je me suis efforcé de trouver des différences caractérisées entre les types locaux de la race indigène. Dans les régions à races mélangées (territoires limitrophes de la Carniole) les caractères morphologiques spécifiques sont assez importants pour différencier 1'abeille indigène de ses voisines italiennes (Apis mellifica var. ligustica SpIN.) et nordique (Apis mellifica var. mellifica L.). Alors que les caractères morphologiques des races européennes sont relativement bien connus, le problème reste entier qui consiste à séparer les écotypes d'une race et le but de nos recherches était d'y introduire plus de clarté.

On utilise pour l'élevage un matériel indigène de départ tel qu'il montre les plus grandes différences génétiques possibles et on multiplie surtout les colonies qui, en tant qu'hybrides " interécotypes " présentent le phénomène de la vigueur hybride. D'après la théorie de VAVıLov, 1'abeille carniolienne autochtone en Yougoslavie, doit montrer un tel polymorphisme. Pour cette raison, nous avions donc quelques chances de succès. Ces chances se trouvent augmentées du fait que l'on se sert de colonies en provenance des zones géographiques les plus éloignées les unes des autres, à l'intérieur de leur territoire. 
Pour le choix des colonies à multiplier plus tard, ou à utiliser dans les stations de fécondation, nous devons toujours tenir compte de la possibilité que la colonie ainsi choisie soit un hybride qui, à cause de l'hétérosis, manifeste des caractéristiques excédant la moyenne (hybride de races ou hybride intérécotypes). Une analyse morphologique différentielle peut nous montrer si les progéniteurs viennent d'une lignée pure ou d'une population à variabilité élevée.

L'importance de telles recherches se conçoit du fait que nous-mêmes, en Slovénie, n'avons jusqu'à présent fait aucune mensuration de notre abeille propre, bien que la Yougoslavie soit reconnue comme la patrie de la carniolienne (KEHRI,E I952; RUTTNER, I952; ZANDER, I953). Dans la seconde moitié du XIX ${ }^{\mathrm{e}}$ siècle, elle s'est étendue de la Slovénie à presque toutes les parties du monde, et à cause de sa grande adaptabilité s'est insérée dans la Trilogie des races d'abeilles standard (carniolienne, italienne, caucasienne) (ABushady, 1948).

Les mesures morphologiques sont également importantes, parce que les chiffres des différents auteurs quant aux mensurations de la Carniolienne diffèrent les uns des autres. Cela tient à ce que certains ont choisi leurs échantillons dans la zone périphérique de l'extension de la race ou dans des territoires nouveaux dans lesquels l'abeille était influencée par des races étrangères. D'autres ont travaillé sur un trop petit nombre d'abeilles, si bien que leurs résultats n'ont pas de fondement statistique.

Comme il s'agit d'une répartition géographique j'ai, par comparaison, évalué les caractères morphologiques d'échantillons venus de territoires où les abeilles nordiques, italiennes et caucasiennes sont répandues.

\section{GÉNÉRALITÉ́S SUR IA MÉTHODE.}

La plupart des échantillons ont été obtenus de zones où les reines sont élevées dans des buts commerciaux. Outre les contrôles réguliers pour vérification de la race, nous nous sommes intéressés à des types locaux de différentes localités de Slovénie et d'autres régions de Yougoslavie. Pour les trois motifs expliqués ci-dessous, nous avons consacré une attention particulière aux frontières albanaises et grecques, ainsi qu'à la Macédoine en général.

Io Dans ces régions de Yougoslavie les plus éloignées de la Slovénie, existe un climat presque subtropical,

$2^{\circ}$ la Macédoine est bien protégée dans sa partie ouest contre les influences étrangères,

$3^{\circ}$ La Macédoine appartient au territoire de la race carniolienne (GoE'TzE, I940) et nous y avons une prépondérance de formes primitives.

Nous pouvons remarquer ici qu'après 1'époque des glaciations, la race carniolienne s'est étendue vers le Nord à partir de ses zones de refuge dans les Balkans (RUTTNER, I952).

Nous avons étudié $5 \mathrm{I}$ ruchers (64 colonies) en Slovénie, I9 en Macédoine (22 colonies), 6 (Io colonies) en différentes parties de la Yougoslavie (Zrenjanin, Beograd, Trebinje, Bileca, Mali Losinj, Tinjan en Istrie).

Nous avons analysé 4 échantillons de races étrangères expédiés de Pologne en I957 (Puck sur la mer Baltique, Skierniewice près de Varsovie, Gorna Niwa près de Pulawy en Pologne centrale et Wroclaw sur 1'Oder). Un autre échantillon nous est venu de Tchécoslovaquie (Dol près de Prague). Les colonies 2 et 7 venant de J.U. 
Belgrade dont les reines ont été importées du Nord de 1'Italie, ainsi que les Abeilles qui accompagnaient les reines de Tbilisi (Russie) appartenaient aussi à des races étrangères.

Nous avons étudié la couleur, les indices alaires et la longueur de la langue. Dans ce travail, nous ne nous sommes intéressés qu'à la coloration.

D'habitude, les abeilles ont été prélevées tard en automne, en hiver ou au premier printemps parmi les mortes des colonies. En Macédoine, nous n'avons étudié que des abeilles d'automne, vivantes. D'après Mrkarıoff (I927) cette période de l'année est la plus convenable pour la prise des échantillons, car d'après cet auteur, les caractères raciaux subissent en été des oscillations très fortes.

Nous avons apprécié les variations des caractères morphologiques par la méthode statistique, en calculant la moyenne arithmétique, la déviation et l'erreur standard. Bien que ces valeurs suffisent à la caractérisation du matériel nous y avons ajouté, particulièrement pour les indices alaires, les valeurs extrêmes. Cela nous permet de comparer les limites de variation des individus isolés avec les données des autres auteurs. On donne aussi dans les tableaux le nombre des abeilles (n) et le mois de la prise d'échantillon.

\section{Nombre des abeilles par échantillon.}

Nous trouvons dans la littérature des données sur la quantité d'abeilles qu'il faut prélever dans une colonie pour les mensurations. GoETzE (I949) pense qu'il faut 60 ouvrières et autant de mâles ; VECCHI et GIAVARINI (I950) utilisent 50 abeilles ; RU'T'NER (I952) étudie au moins 50 abeilles par colonie mais dans la plupart des cas, au moins Ioo abeilles et mâles. Soose (I954) en prélève régulièrement 250 ; 1'Institut polonais (Bornus et Demianowicz, I957 renseignement oral) étudie roo abeilles.

\section{RE,CHERCHES SUR LA COLORATION.}

On ne doit pas s'étonner de ce que l'estimation de la couleur ait été tellement employée par les apiculteurs Slovènes dans le tri des races. La couleur des premiers tergites abdominaux saute aux yeux et JANscha (I734-I773) l'utilisait déjà dans sa description des abeilles. A l'époque des exportations qui commencèrent dans la deuxième moitié du xIx ${ }^{\mathrm{e}}$ siècle, et se firent en Slovénie (Carniole) avec des abeilles vivantes vendues en gros, beaucoup de négociants éliminèrent les reines issues des colonies qui portaient des marques jaunes; ce moyen de les séparer a pu avoir sa valeur jusqu'il y a dix ans quand VERBIC (I947) établit les bases de l'élevage des reines, bien que GoETZE (I940) n'aie trouvé aucune régularité de couleur dans les races des Balkans ou de l'Italie. Comme pour nos éleveurs, la couleur est aussi pour les auteurs étrangers un caractère racial valable (ABUSHADY I948). Dans nos propres recherches, nous avons essayé de distinguer dans quelle mesure la couleur des premiers tergites abdominaux pouvait servir de caractère spécifique pour la carniolienne et pour ses écotypes purs, malgré l'opinion de Ru'rrNER (I952) selon laquelle la couleur des abeilles et des mâles (Gravarini I953) ne peut être employée pour caractériser les races d'abeilles européennes.

Méthode. - En I955 (RImAR, I956) nous avons utilisé pour l'étude de la coloration des abeilles, la méthode de GOE'TzE (I949) en vue de l'attribution des notes pour la sélection. Mais à cause de ses défauts, nous 1'avons remplacée les années suivantes 
par l'échelle de 9 degrés du même auteur (GOETzE I940). Comme les principales déterminations des couleurs effectuées par les auteurs l'ont été sur des souches yougoslaves, nous avons considéré comme acquis qu'il était convenable de préférer aux fins de comparaisons, l'échelle de GOETZE à celles des autres auteurs (ARMBruster I923, SoOSE, I954).

D'après la longueur et la largeur de la bande claire du second tergite abdominal on peut répartir nos abeilles en 9 classes (fig. I) :

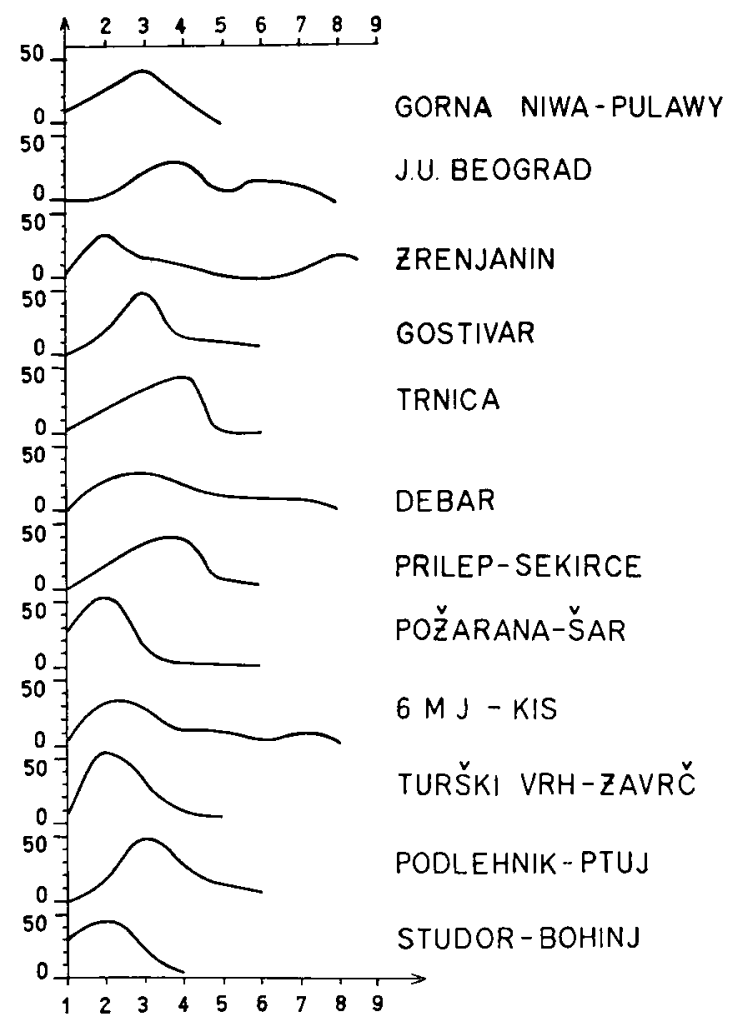

FIG. r. - Variabilité de la couleur ches certains échantillons.

En ordonnées, le nombre d'Abeilles.

En abscisses, les classes de coloration du second tergite.

Classe I: Abeilles à dos complètement sombre. Bord postérieur des tergites foncé. L'intensité de la coloration dans les classes 2 à 9 est caractérisée comme suit :

Classe 2: Une petite ligne claire sur tout le tergite.

Classe 3: Petite ligne avec des petits angles.

Classe 4: Ligne à angles plus larges.

Classe 5: Petites taches allongées dans le sens de la longueur.

Classe 6: Taches plus volumineuses.

Classe 7: Une petite bande claire sur tous les tergites.

Classe 8: Bande plus large et plus claire.

Classe 9: Bande claire très large. 
On verra les résultats dans les tableaux I à 5 .

TABLEAU I

Comparaison d'après la couleur des Abeilles de souche slovène.

\begin{tabular}{|c|c|c|c|c|c|c|}
\hline Numéro & Origine & $\begin{array}{l}\text { Mois du } \\
\text { prólèvement }\end{array}$ & n & Moyenne et écart & $\begin{array}{l}\text { Erreur } \\
\text { standard }\end{array}$ & Observations \\
\hline $1 \ldots$ & 13. A., Golobrdo-Medvode & II & 150 & $3,2: 00$-1 1,8370 & 1,3549 & \\
\hline$\because \ldots$ & S. J., Studor-13ohinj & III & 150 & $1, \times 66 ; \doteq 0,5458$ & 0,7309 & \\
\hline $3 \ldots \ldots$ & 11. J., Iodlehnik-Ptuj & III & 60 & 2,$266 ; \quad 1.1,3175$ & 1,1478 & \\
\hline 1...... & 3. J., Rače-Haribor & III & 60 & $2,133+0,7276$ & 0,7529 & \\
\hline $5 \ldots \ldots$ & S. J., Blejska Dobrava & III & (;0) & $-2,16 f=-2,2547$ & 1,5015 & \\
\hline (i.... & 13. N., Hardek-Ormož & III & bo & $2,350 \pm 1,819^{\prime}$ & 1,3488 & \\
\hline $7 \ldots$ & T'. I., Grškovje-I'odlehnik & IV & 60 & $-, 683 \pm 1,3726$ & $1,171 \mathrm{t}$ & \\
\hline x... & Si. J., Turski-vrh-Zavrě & III & 80 & $2,212 \pm 0,7011$ & 0,8373 & \\
\hline $9 .$. & (S. T., Irenovice-Postojna & IV & 60 & $2,750 \doteq 2,2567$ & $1,502: 2$ & \\
\hline $10 .$. & P. J., Savica-Bohinj & IV & 60 & $2,300 \div 1,1627$ & 1,0782 & \\
\hline $11 .$. & 11. A., Jezero-Trebnje & III & $1 ; 0$ & $2,400 \pm 2,1500$ & 1,46002 & \\
\hline $1: \ldots$ & 11. A., Jezero-Irebnje & IV & 100 & $3,: 10=1,1978$ & $1,09 \%$ & 2 Colones \\
\hline $13 \ldots$ & J. K., Velika Iolia & II I & 100 & $2, x, 50+1,969$ & 1,3953 & \\
\hline $11 \ldots$ & I. M., Velika I,oka & III & 100 & $2,870 \doteq 1,0233$ & 1,0115 & \\
\hline $15 \ldots$ & $\therefore$ J., Velika Loka & $V$ & (if) & $2,600=1,36 ; 27$ & 1,1673 & \\
\hline $16 .$. & $\begin{array}{l}\text { Sitation de fécondation Soteska- } \\
\text { Bled }\end{array}$ & VII & 100 & $1,980=0,6166$ & $0,80 \div 1$ & Voir Nr. 2 \\
\hline
\end{tabular}

TABLEAU 2

Comparaison d'après la conleni' des Abeilles de souche Macílonienne.

\begin{tabular}{|c|c|c|c|c|c|c|}
\hline Niméro & Origine & $\begin{array}{c}\text { Mois dut } \\
\text { P'ćlevement }\end{array}$ & 11 & Moyenne et coart & $\begin{array}{l}\text { Erreur } \\
\text { standard }\end{array}$ & Remarques \\
\hline $1 \ldots$ & (. D., Trubarevo-skopje & $X$ & 100 & $2,6000 \quad \therefore \quad 1,0303$ & 1,0150 & \\
\hline$\because \ldots$ & P. 7., Radoviste & $\mathrm{X}$ & 100 & $3,010 \doteq 1,0201$ & 1,0100 & \\
\hline $3 \ldots \ldots$ & J. P., Skopje & $\mathrm{X}$ & 75 & $2,520 \pm 0,8209$ & 0,9060 & \\
\hline $4 \ldots \ldots$ & J. B., Gostivar-Cajlije & $\mathrm{X}$ & 100 & $2,690 \doteq 0,9802$ & 0,9899 & \\
\hline $5 \ldots \ldots$ & S. J., Crostivar & $\mathrm{X}$ & 1001 & $3,150 \pm 1,3207$ & $1,11 \%$ & \\
\hline ii...... & L. М., Pozarana & $X$ & 100 & $2,030 \doteq 0,8373$ & 0,9150 & \\
\hline $7 \ldots \ldots$ & Ingr. M., Gostivar & $\mathrm{X}$ & 1010 & $3,370 \pm 1,2,555$ & 1,1160 & \\
\hline $8 \ldots$. & s. r. z., Tresonce & $\mathrm{X}$ & 100 & $3,060 \doteq 0,9051$ & 0,9509 & \\
\hline$\because \ldots$ & Manrovo-Trnica & $X$ & 100 & $3,260 \pm 0,8408$ & 0,9169 & \\
\hline $10 \ldots$ & I. I'., Debar & $\mathrm{X}$ & 100 & $3, \times 70) \therefore 2,7001$ & $1,0,432$ & \\
\hline $11 \ldots$ & F. A., Ohrid & $\mathrm{X}$ & 70 & $3,,_{1}^{\prime}(0) \doteq 0,7652$ & 0,8718 & \\
\hline $12 \ldots \ldots$ & B., Trebeniste & $\mathrm{X}$ & 100 & $3,800 \div 1,3131$ & 0,1159 & \\
\hline $13 \ldots \ldots$ & Pecl. st. Bitola & $\mathrm{X}$ & 100 & $3,310 \div 1,5291$ & 1,2365 & \\
\hline $1 ! \ldots \ldots$ & l'čl. st. Prilep-Sekirce & $\mathrm{X}$ & 100 & $3,360=0,77 \times 1$ & $0, \times 8 \times 10$ & \\
\hline $15 \ldots \ldots$ & P'cel. st. Prilej-Borotino & $\mathrm{X}$ & 100 & $3,200 \pm 0,7 \times 78$ & 0,8875 & \\
\hline $11 \ldots \ldots$ & D. J., Gergelija & $x$ & 100 & $3,470 \therefore 0,7800$ & $0, \$ 8 \times 37$ & \\
\hline $17 \ldots$ & P. R., Gevgelija & $\mathrm{X}$ & $\because 0$ & $1, \because 50 \doteq 0,9336$ & 0,96663 & \\
\hline $18 \ldots$ & Polj. stop. Dojran & $\mathrm{X}$ & 100 & 3,110 上 0,1665 & 0,6837 & \\
\hline
\end{tabular}

Les échantillons étudiés dans le tableau I nous ont été envoyés en 1956 par des éleveurs de reines de tous les territoires de Slovénie. Les no 2 et 16 correspondent à deux colonies du même rucher de caractères extraordinairement voisins. La couleur 
de toutes les colonies slovènes est comprise entre la seconde et la troisième classe de l'échelle (moyenne 2,538).

Le tableau 2 groupe les échantillons rapportés d'un voyage en Macédoine en $\mathbf{I} 956$; les $\mathbf{n}^{\mathbf{0}} 4$, I4 et I 5 correspondent à des élevages maintenus, à ce qu'on nous a expliqué, depuis 300 ans dans le même village. Le $n^{0}$ Io allait butiner les champs de fleurs albanais, les $n^{\circ}$ I 6 et I7, les champs grecs. L'échantillon de DEBAR (no Io) est caractérisé par une grande variabilité. La couleur du deuxième tergite abdominal est dans 1'ensemble assez homogène. Le no 6 de Pozarana sur le Sar montre le plus faible degré de pigmentation (moyenne de tous les échantillons, 3,205).

Les données du tableau 3 sont caractérisées par la plus grande hétérogénéité. Le $\mathrm{n}^{0}$ I peut être considéré comme représentant, ce qu'on appelle la "souche du Banat "; dans aucun autre échantillon nous ne retrouvons d'aussi larges variations ; L'échantillon no 8 vient d'Istrie, le $n^{\circ} 7$ des Iles Dalmates, provinces qui se sont trouvées toutes les deux jusqu'en I 945 sous la dominance italienne. On peut considérer comme représentant 'd'Apis mellifica var. ligustica SpIN, les $\mathrm{n}^{\circ} 2$ et 6 dont les reines ont été importées directement des environs de Bologne.

TABLEAU 3

Comparaison d'après la couleur d'Abeilles yougoslaves de différentes origines.

\begin{tabular}{|c|c|c|c|c|c|c|}
\hline Numéro & Origine & $\begin{array}{c}\text { Mois du } \\
\text { prélèvement }\end{array}$ & n & Moyenne et écart & $\begin{array}{l}\text { Erreur } \\
\text { standard }\end{array}$ & Observations \\
\hline$\ldots$ & J. S., Zrenjanin & $\mathrm{X}$ & 100 & $4,290 \pm 6,8544$ & 2,6180 & Colonie No $^{\circ}$ \\
\hline$\cdots$ & J. U., Beograd & Il & 90 & 4,355 上 1,9845 & 1,1087 & 2 \\
\hline 3. & J. U., Beograd & II & 90 & $3,255 \pm 1,2935$ & 1,1373 & 14 \\
\hline 4... & J. I., Beograd & II & 90 & 2,988 is 1,4992 & $1, \geq 122$ & 15 \\
\hline $5 \ldots$ & J. II, Beograd & II & 50 & $3,6,60 \pm 2,7922$ & 1,6709 & $2: 2$ \\
\hline 6... & J. U., Beograd & II & 100 & $4,6 \pm 0+2,2581$ & 1,5026 & 7 \\
\hline $7 \ldots$ & M. Losinj-Zale & VII & 70 & $3,11:=0,8 \geq 77$ & 0,9098 & \\
\hline $8 \ldots$ & B. J., Brečevici-Ťnjan & IV & (i) & $2,650 \pm 2,8415$ & 1,$1 ; 0 \times 7$ & \\
\hline
\end{tabular}

Dans la table 4 , les $\mathrm{n}^{\circ} \mathrm{I}-4$ viennent du territoire de $1^{\prime} A$ pis mellifica var. mellifica $L$. en Pologne. Le $n^{\circ} 5$ est la "souche Dol" de 1'Institut d'Apiculture de Prague qui est assez homogène; mais le nombre des abeilles de l'échantillon est faible. L,e $n^{\circ} 6$ appartient vraisemblablement à une souche caucasienne.

TABLEAU 4

Comparaison d'après la couleur d'abeilles polonaises, tchèques ou soviétiques.

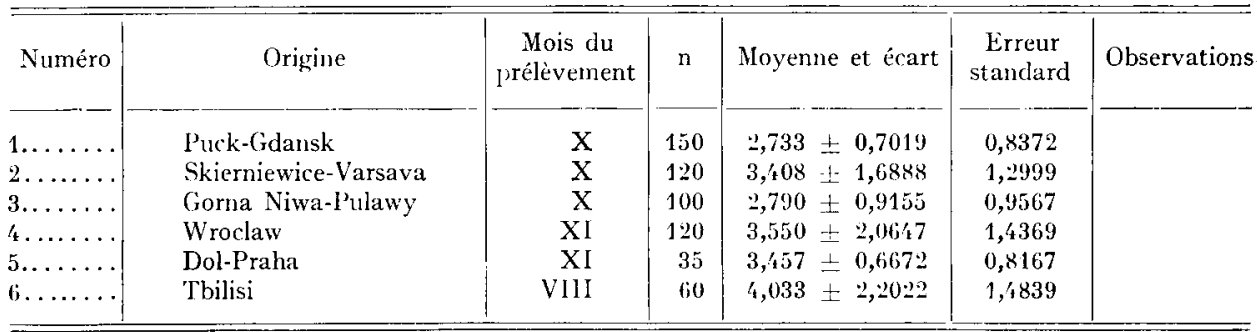


Dans le tableau 5 sont comprises 2 colonies du rucher de 1'Institut de Ljubljana.

TABLEAU 5

Variation de la couleur de deux colonies du rucher de l'Institut pendant les mois de mars à juillet.

\begin{tabular}{|c|c|c|c|c|c|c|}
\hline Numéro & Origine & $\begin{array}{c}\text { Date du } \\
\text { prélèvement }\end{array}$ & $\mathrm{n}$ & Moyenne et écart & $\begin{array}{l}\text { Erreur } \\
\text { standard }\end{array}$ & Observations \\
\hline 1.. & KIS - 12 J. K. & 11.3. & 100 & $2,850+1,9469$ & 1,3953 & \\
\hline$\because$. & $\mathrm{KIS}-12 \mathrm{~J} . \mathrm{K}$. & 11. & 100 & $2,770 \div 2,8051$ & $1,6,7,8$ & \\
\hline 3.. & $\mathrm{KIS}-12 \mathrm{~J} . \mathrm{K}$. & 11. 5. & 100 & $2,950 \pm 2,9772$ & $1,725 /$ & \\
\hline $4 .$. & $\mathrm{KIS}-12 \mathrm{~J} . \mathrm{K}$. & 11. fi. & 100 & $2,840 \div 1,9539$ & $1,397 \mathrm{x}$ & \\
\hline 5. & $\mathrm{KIS}-12 \mathrm{~J} . \mathrm{K}$. & 11. 7. & 100 & $2,850 \therefore 2,0075$ & 1,1168 & \\
\hline b......... & KIS - $6 \mathrm{~J} . \mathrm{M}$. & 11.3. & 100 & $2,870+1,0233$ & 1,0115 & \\
\hline $7 \ldots \ldots$ & KIS - ; J. M. & 11. 亿. & 100 & $2,820 \pm 1,0985$ & 1,0480 & \\
\hline $8 \ldots \ldots$ & KIS -, $\mathrm{J} . \mathrm{M}$. & 11.5. & 100 & $2,920+1,0642$ & 1,0316 & \\
\hline $9 \ldots \ldots$ & KIS - $6 \mathrm{~J} . \mathrm{M}$. & 11. 6. & 100 & $3,440 \div 2,7337$ & 1,65333 & \\
\hline $10 \ldots \ldots$ & KIS - $\quad$ J J. M. & 11. 7. & 100 & $2,750 \quad \therefore \quad 1,0833$ & 1,2582 & \\
\hline
\end{tabular}

\section{DISCUSSION}

La pigmentation du second tergite abdominal de l'Abeille carnolienne de Yougoslavie s'élève en moyenne de 0,667 depuis le Nord de la Slovénie jusqu'au SudOuest de la Macédoine. La couleur la plus foncée est trouvée pour la Slovénie à Studor-Bohinj, pour la Macédoine à Pozarana-Sar. I1 est étonnant de constater 1'homozygotie de la souche Studor (dispersion $0,5458-0,6466$ ) qui en I957 a été utilisée dans les ruchers de fécondation de Voje-Bohinj, Soteska-Bled et Jurgovo sur Pohorje.

Les observations sur les Abeilles de Lika et d'Herzegowina permettent de penser que les Abeilles yougoslaves de la ligne montagneuse Triglav (Alpes Juliennes) Ohrid sont les moins pigmentées. La couleur est plus claire dans les plaines du Frioul, sur les mers adriatiques et Égée et sur la plaine Panonnienne.

Il faut signaler le travail de Mutoo (I956) qui a trouvé que dans 1'Inde, les abeilles des montagnes sont grises ou noires, alors que celles des plaines sont jaunâtres. TARANov dit aussi que le jaune caractérise plutôt les abeilles du Sud. Les échantillons yougoslaves les plus foncés trouvés à Bohinj et Sar montrent la même tendance (fig. 2).

On doit remarquer qu'en Slovénie, la pigmentation du second tergite abdominal est en moyenne un peu moins foncée que ne le montrent les échantillons. Les échantillons viennent d'élevages de reines dont on élimine sans arrêt les reines à dessins jaunes et de zones où les ruches sont peu déplacées.

Nos recherches ont confirmé nos travaux précédents (RIHAR - I956) selon lequel on ne peut trouver en Yougoslavie d'abeilles foncées sans marques jaunes. On y voit en général de grosses différences de coloration (Bohinj I,866 - Zrenjanin 4,29o). Parmi les échantillons de valeur moyenne aussi élevée que 4,o on peut conclure avec certitude à une influence de ligustica (tableau $3 \mathrm{n}^{\circ} \mathrm{I}$ ) où à une véritable importation de ligustica (tableau $3 \mathrm{n}^{0} 2$ et 6 ).

Nos recherches montrent qu'en Yougoslavie l'Abeille carnolienne se présente 


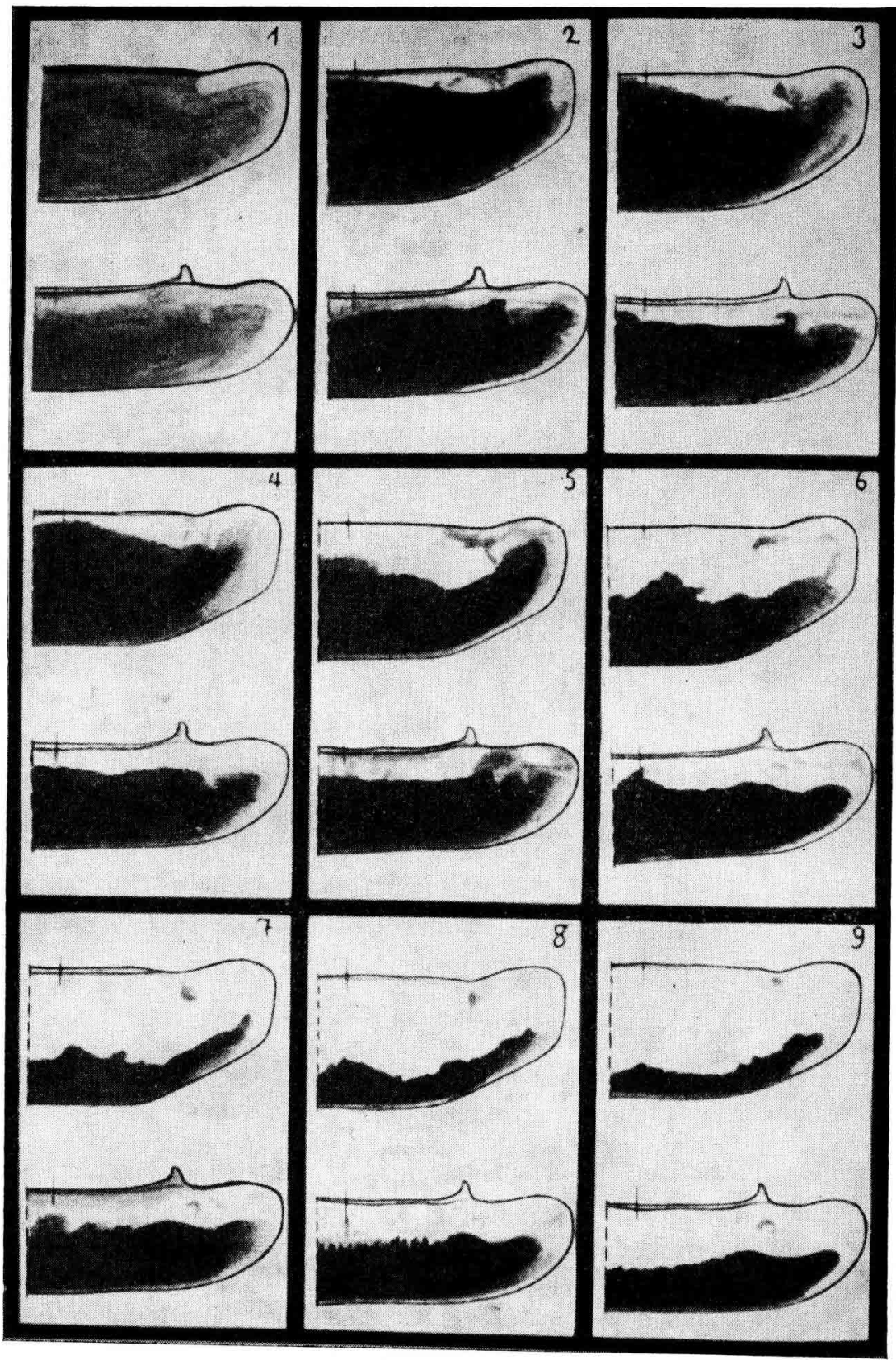

FIG. 2. - Echelle de la coloration des $2^{\mathrm{e}}$ et $3^{\mathrm{e}}$ tergites ches l'Abeille (d'après GOETZE). 
avec une pigmentation foncée, dans un territoire où les caractéristiques de coloration sont en général celles des races du Nord. On ne trouve dans aucune race l'Abeille foncée idéale. D'après cela on ne doit pas s'appesantir sur les irrégularités consistant par exemple en des marques jaunes sur le deuxième tergite abdominal. Il n'y a pas de raison d'admettre l'hypothèse suivant laquelle la carnolienne viendrait d'un croisement très ancien entre mellifica et liqustica (Gravariñ, I953). Cette possibilité est à exclure à mon sens quand on considère les moyennes et les écarts des échantillons sur la ligne Triglav-Ohrid. On pourrait plutôt admettre que l'Abeille carnolienne a trouvé asile en Macédoine pendant la période glaciaire et s'est répandue plus tard plus au Nord. Les données de RUTT'Ner (I950) suivant lesquelles il s'agit d'une race autochtone, qui s'est repliée dans les Balkans et plus tard a émigré dans le Nord peuvent être complétées. Pendant la période glaciaire c'était la Macédoine qui constituait la patrie de ces Abeilles; dans les territoires plus au sud il s'agit d'Abeilles grecques (GOETZE - I940), qui sont d'après MUI,LER (I940) très hétérogènes.

Les différences de coloration entre les Abeilles carnoliennes et italiennes ne sont pas seulement quantitatives, mais qualitatives : chez les italiennes la couleur jaune rappelle celle du cuir, chez les carnoliennes elle tire vers le rouge brun; JANScha l'avait remarqué déjà qui parle de tergites jaune-rouges.

Il faut remarquer que tous les échantillons de la race sombre du Nord (Mellifica) sont plus fortement pigmentés que les Slovènes. Fin général les Abeilles de la Baltique ne se différencient pas de celles de la Macédoine. Il est évident que les caractéristiques de coloration ne dépendent que dans les limites restreintes de la position géographique (Mackensen, RutTner - I952). Goetze (I940) fournit des données sur les variations larges et irrégulières de la couleur des races du Nord. Pour la souche " Nigra " la plus pure des races du Nord, on trouve des valeurs de I,7336 (Suisse) à $2,835^{8}$ (Erlangen) ; pour d'autres races les valeurs vont jusqu'à 4,3. D'après le même auteur les valeurs régulièrement plus hautes pour l'italienne sont de 4,o mais dans le sud montent à 7,2 et 8,9 .

ROBERTS (I95I) a montré qu'il est difficile de distinguer des races d'après la couleur du tergite abdominal : à l'aide de croisements interraciaux on peut montrer en effet que la couleur de 1'abdomen dépend d'au moins 7 gènes différents. MACKENSEN (I956) a réalisé par homozygotie la couleur idéale de la carnolienne foncée ou de 1'Abeille italienne. Mais leurs lignées pures se distinguaient par leur manque de vitalité et ne pouvaient se maintenir d'elles-mêmes, ce qui met en question les suites d'un élevage poursuivi dans cette direction. On ne doit pas pourtant passer sous silence les données d'ABUSHADy (I948) d'après lequel la carnolienne est d'autant plus féconde qu'elle est plus foncée. Selon toute vraisemblance les écotypes de la carnolienne sélectionnés d'après la couleur sont très recherchés par les apiculteurs et dans divers pays (Égypte par ex.) où on les croise avec la race indigène pour obtenir la luxuriance de la génération $F_{1}$. Cela explique cette sélection d'après la couleur, à laquelle nos éleveurs sont fortement attachés. A ce propos il faut dire aussi un mot de la variation de la couleur suivant la saison (MULiLER I940 - PIRNAT I954). D'après la méthode cidessus indiquée nous avons suivi la couleur de deux colonies pendant les 5 mois de mars à juillet qui montrent les plus fortes oscillations thermiques (voir table 5). Les moyennes et l'écart des échantillons " I2 J. K. " sont nettement apparentés à ceux des échantillons " 6 J.M. " dont les valeurs sont spécialement hautes en juin. Le phénomène de l'éclaircissement des Abeilles d'été doit s'expliquer par la polyan- 
drie des reines (ROBERTS I942 - 'TRIASKO I95I - RUTTNER I954 - PEER I956). Comme les spermatozoïdes des différents mâles ne se mêlent pas dans la spermathéque, on peut trouver en des périodes bien déterminées dans le temps des Abeilles différemment colorées.

\section{CONCIUSIONS.}

I $^{\mathbf{0}}$ Les échantillons des carnoliennes et de toutes les autres races européennes montrent des marques jaunes sur le $2^{\mathrm{e}}$ tergite abdominal.

$2^{\circ}$ Les moins pigmentées sont les Abeilles de Bohinj en Slovénie et de Pozarana en Macédoine.

$3^{\circ}$ A partir des montagnes yougoslaves où l'on trouve les Abeilles les plus foncées on remarque en allant vers les plaines et les mers une augmentation de la pigmentation.

$4^{\circ}$ A l'aide de la couleur du $2^{\mathrm{e}}$ tergite abdominal on peut différencier facilement la carnolienne de ligustica, mais non de mellifica.

$5^{\circ}$ Quelques écotypes de la carnolienne petrvent se distinguer d'après la couleur. Dans le cadre des variations de la race l'emploi traditionnel des marques colorées comme moyen de sélection massale est toujours justifiable.

$6^{\circ}$ Les échantillons dont la moyenne atteint 2,5 peuvent être considérés comme représentant le champ de variation le plus étroit de la race carnolienne et ceux dont la moyenne atteint 3,2 comme le plus large.

$7^{0}$ Les variations de température saisonnières n'ont pas d'influence sur la couleur du second tergite abdominal.

Reçu pour publication en septembre 1960

\section{RÉFÉRENCES BIBLIOGRAPHIQUES.}

Armbruster L., 1923. Wie untersucht man Bienenstămme und Bienenkreuzungen auf ihre Farbe? Arch. 1. Bienenk., V, $97-\mathrm{I} 3 \mathrm{O}$.

Giavarini J., I953. Ricerche sui caratteri razziali dell'Apis mellifica ligustica SpIN. Men. Soc. Entom. Italiana, XXXII, I $19-128$.

GiavaRinI J., Vecchi A., 1950. Ricerche sui caratteri razziali dell'ape domestica. Boll. Ist. Entom

Univ., Bologna, 18.

GoETzE G., I940. Die beste Biene, 200 p. Liedloff, Loth et Michaelis, Leipzig.

GoETZE, G., 1949. Imkerliche Züchtungspraxis, Nlannover.

Grout, 1948. The Hive and the Honey, Hamilton.

JAnscha A., I775. Die vollkommene Lehre von der Bienenzucht, Wien.

KEHRLE A., I95I. In search of the best strains of bees. Bee World, 32, (7), 49-52 (8), 57-62.

Mackensen O., 1956. Einige Wirkugen der Inzucht bei den Honigbienen, XVI. Cong. Intern. Apic., Vienne Michailoff A. S., 1927. Uber die Saison - Variäbilitat der Honigbiene. Arch. Bienenk., VIII, 304-31 I.

Muller K., 1939. Studien zur Rassenkenntnis der Ilonigbiene. Deutsch. Imkert., 13, I89-191, $207^{-210 .}$

PEer D. F., I956. Multiple Mating of Quuen Ioneybees. 7. Econ. Entom., 49, (6) 74I-743.

Pirnat J., I954. Se o cebelah z rumenimi obrocki. Slov, cebelar. 56. I9-20.

Rihar J., I956. Porocilo Kmetijskega instituta Slovenije o delu v cebelarstvu za leto 1955. Slov. Cebela, 58, 2 I I-21 5, 250-264.

RIHAR J., 1958. Biometrische Untersuchungen der Bienen in Jugoslawien, XVII Cong. intern. Apic. Rome. RoJEC V., I944. Barva in velikost cebel v luci splosne menjavosti. Ceb. zbornik.

Ruttner F., 1952. Die Aussenmerkmale des Carnica -- Stammes Troisek. Oster. Imker, 2 (4), 67-69.

Ruttner F., Mackensen O., I 954. The genetics of the Honeybee. Bee World. 33, (4), 53-62, 33, (5), 7 I-79.

Soose E., 1954. Einfluss der Temperatur auf die Ausgestaltung von Flügelindex und Panzerfarbe der

Honigbliene. Archiv. f. Bienenk., 31 (2), 49-66.

Triasko V. V., I95I. Priznaki osemenenosti pcelinih matok, Pchelovosdstvo, 11, 25-31.

Worke J., I955. Mnogokratnoe sparivanie pcelinoi matki. Apis mellifica. $\mathrm{L}$. vo vremja odnogo bracnogo vileta. Blull. pol. Akad. nauk., III, (5), I77-182.

VERBIC J., I947. Vzrejajmo najboljse cebele, Ljubljana.

ZANDER E., I953. Die Zucht der Biene. Stuttgart. 